\title{
Unraveling the Thrill of Metric Image Spaces
}

\author{
Patrik Eklund and Fredrik Georgsson \\ Umeå University, Department of Computing Science, SE-901 87 UMEÅ, Sweden, \\ \{peklund,fredrikg\}@cs.umu.se, \\ WWW home page: http://www.cs.umu.se/ ${ }^{\sim}$ peklund,fredrikg\}
}

\begin{abstract}
In this paper we focus on distances between textures, and develop metrics on image spaces in contexts of image transformations. Given a metric on the range space, we can generate the initial topology for the domain space. For this topology we can obtain a corresponding metric using well-known metrization constructions, also providing granularity of the metrics. Examples are drawn from the Spatial Gray Level Dependency (SGLD) transformation and the application domain is texture recognition in medical imaging.
\end{abstract}

\section{Introduction}

In recent years there have been several attempts to define metrics for measuring distances between images or components in images. Inspired by [1], with questions related to finding appropriate topologies for morphological studies, it seems similarly natural to seek for appropriate metrics for particular image analysis and computer vision problems. These approaches are often focused on overcoming problems related to distortion given a domain space of usually greyscaled images. A typical solution is given in [2], extending techniques originally proposed by [1], which builds upon Hausdorff metrics on classes of compact sets. Robustness of metrics is studied with respect to distortions. Another approach is provided in [3], where neighborhoods and weightings are used to define classes of semi-pseudo metric spaces. The triangular inequality is sacrificed, and results relate to how neighborhood based metrics shift to weighting based metrics in the limit.

In this paper we focus on distances between textures, and suggest to derive a metric using observations on transformations of images. Thus we develop metrics on image spaces in contexts of image transformations, and therefore consider both the original domain space as well as the range space of the image transformation. For the generated metric on our domain space we obviously require that nearness of a pair of images in the range space reflects nearness of the original images in the domain space. This is to say that we require our image transformation to be a continuous mapping. Further we desire to have a certain granulation in our metric, i.e. we want the metric to be parameterized in some appropriate way. The construction stems from our starting point that Euclidean distances or Hausdorff metrics are inappropriate in a domain space but natural to consider for the range space. Thus given a suitable metric on the range space, 
we have the corresponding topology and can generate the initial topology for the domain space. If this initial topology is reasonably well-equipped we can obtain a corresponding metric using well-known topological metrization constructions. These constructions also provide the granulation parameters. The construction also relate to our cognitive notion of distances between textures.

Our exemplifications are drawn from Spatial Gray Level Dependency (SGLD) transformation [4] and the application domain inspiring these developments is texture recognition in mammographic images 56 . SGLD transformations have been successfully used also for other imaging problems [7].

\section{Notation and Basic Constructions}

Let $X$ denote a domain space of images, and let $L$ be a set of 'pixel values'. Thus an image is a function $f: X \rightarrow L$. The space, or set, of such images, or functions, is denoted $F(X, L)$. An image transformation $\tau$ is a mapping between image spaces, i.e. $\tau: F(X, L) \rightarrow F(Y, M)$. In a 2-dimensional grey-scale image we would have $X=A \times B$ and $L$ would be a linearly ordered complete lattice.

For our formal constructions we obviously need to consider underlying measure spaces and corresponding integrability of images as functions. However, our constructions are based on a rather informal mathematical treatment, and therefore these considerations are left out. When restricted to the real-valued case, the constructions can be seen to build upon Lebesque measures and integrability. For further background to topologies and (pseudo-)metrics, see e.g. 8].

The pointwise defined metric $d_{p}$ on $F(X, L)$ is given by

$$
d_{p}\left(f_{1}, f_{2}\right)=\left(\int_{X}\left|f_{1}-f_{2}\right|^{p}\right)^{1 / p} .
$$

The typical example demonstrating the drawback of this metric is the following. Let $X=A \times B$, where $A=\left\{a_{1}, \ldots, a_{m}\right\}$ and $B=\left\{b_{1}, \ldots, b_{n}\right\}$, i.e. $X$ be a grid of pixels, and further $f_{1}: X \rightarrow[0,1]$ be defined by

$$
f_{1}\left(a_{i}, b_{j}\right)=\left\{\begin{array}{l}
1, \text { if } i+j \text { is even, } \\
0, \text { otherwise }
\end{array}\right.
$$

and $f_{2}: X \rightarrow[0,1]$ be defined by

$$
f_{2}\left(a_{i}, b_{j}\right)=\left\{\begin{array}{l}
1, \text { if } i+j \text { is odd } \\
0, \text { otherwise }
\end{array}\right.
$$

i.e. $f_{1}$ and $f_{2}$ represents $m \times n$ 'chessboards' with, respectively, white and black in the upper left corner, see Figure 1.

Then $\left(\int_{X}\left|f_{1}-f_{2}\right|^{p}\right)^{1 / p}=\left(\int_{X} \mathbf{1}\right)^{1 / p}$, which clearly is undesirable. In order to introduce metrics generated using transformations, consider first the mapping $f: X \rightarrow Y$, and a (pseudo-)metric $(Y, e)$. Then the mapping $d: X \times X$ defined by 

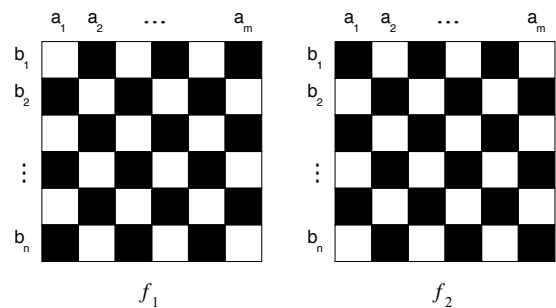

Fig. 1. Chessboard texture

$d\left(x_{1}, x_{2}\right)=e\left(f\left(x_{1}\right), f\left(x_{2}\right)\right)$ is a (pseudo-)metric for $X$, and $f:(X, \mathcal{T}) \rightarrow(Y, \mathcal{U})$ is continuous, where $\mathcal{T}$ and $\mathcal{U}$ are the corresponding metric topologies generated by $d$ and $e$, respectively.

Note that these constructions do not involve any granulations. Usually, some granulation features are desirable from application point of view. Granulation can be achieved either using neighbourhoods of points in $X$, or, as mentioned above, using metrization techniques related to the initial topology on the domain space given by the metric topology on the target space. Details are presented in the following section.

\section{$3 \quad$ Metric Image Spaces}

In this section we will construct metrics on $F(X, L)$, given image transformations

$$
\tau: F(X, L) \rightarrow F(Y, M)
$$

and metrics on $F(Y, M)$. Our constraint to have nearness of images $f_{i} \in F(X, L)$ being related to nearness of corresponding transformed images $\tau\left(f_{i}\right) \in F(Y, M)$ will be guaranteed by the requirement that $\tau$ is continuous.

Throughout this section we will assume $F(Y, M)$ to be equipped with some $L^{p}$ metric

$$
e_{p}\left(g_{1}, g_{2}\right)=\left(\int_{Y}\left|g_{1}-g_{2}\right|^{p}\right)^{1 / p}
$$

\subsection{Granulation Using Neighborhoods}

Assume that $Y$ has an underlying metric. For $g \in F(Y, M)$, let $\kappa^{(\gamma)}: F(Y, M) \rightarrow$ $F(Y, M)$ provide a modification of $g$ so that $\kappa^{(\gamma)}(g)(y)$ combines values of $g\left(y^{\prime}\right)$, $y^{\prime} \in B(y, \gamma)$, in some appropriate way.

A particular choice for $\kappa$ might be

$$
\kappa^{(\gamma)}(g)(y)=\frac{\int_{B\left(y^{\prime}, \gamma\right)} y^{\prime} \cdot g}{\int_{B\left(y^{\prime}, \gamma\right)} y^{\prime}},
$$


i.e. the mean value of the neighborhood. This a low-pass filtering of $g$, which means that high frequencies will be suppressed in the metric. This in turn means that we obtain a metric that is relatively robust to noise.

Another choice of $\kappa$ is

$$
\kappa^{(\gamma)}(g)(y)=\sup _{y^{\prime} \in B(y, \gamma)} g\left(y^{\prime}\right) .
$$

Taking the supremum of the neighborhood might in some applications be motivated. This is mainly true if our $\tau$ has a very good locality, that is, it concentrates $\tau(f)$ to some points in $Y$. In these cases it is not desirable to smear these points by using a mean value.

We can now define a metric on $F(X, L)$ through

$$
c^{(\gamma)}\left(f_{1}, f_{2}\right)=\left(\int_{Y}\left|\kappa^{(\gamma)}\left(\tau\left(f_{1}\right)\right)-\kappa^{(\gamma)}\left(\tau\left(f_{2}\right)\right)\right|^{p}\right)^{1 / p} .
$$

We will use $c^{(0)}\left(f_{1}, f_{2}\right)$ to mean $\left(\int_{Y}\left|\tau\left(f_{1}\right)-\tau\left(f_{2}\right)\right|^{p}\right)^{1 / p}$. A normalization of $c$ can be given as

$$
\hat{c}^{(\gamma)}\left(f_{1}, f_{2}\right)=\frac{c^{(\gamma)}\left(f_{1}, f_{2}\right)}{\left(\int_{Y} \mathbf{1}\right)^{1 / p}} .
$$

Constructions in [3] correspond to using $\inf _{y_{1}, y_{2} \in B(y, \gamma)}\left|\tau\left(f_{1}\right)\left(y_{1}\right)-\tau\left(f_{2}\right)\left(y_{2}\right)\right|$, as compared to our $\left|\kappa^{(\gamma)}\left(\tau\left(f_{1}\right)\right)(y)-\kappa^{(\gamma)}\left(\tau\left(f_{2}\right)\right)(y)\right|$. We have that $\gamma>0$ and

$$
c^{(0)}\left(f_{1}, f_{2}\right)=d_{\infty}\left(\tau\left(f_{1}\right), \tau\left(f_{2}\right)\right)
$$

since $\lim _{p \rightarrow \infty} d_{p}\left(f_{1}, f_{2}\right)=\sup \left(f_{1}, f_{2}\right)$ Note that this holds regardless of the $\kappa^{(\gamma)}$ used.

Similarly, $\gamma \leq d(Y)=\sup \left\{d\left(y_{1}, y_{2}\right): y_{1}, y_{2} \in Y\right\}$ and

$$
\begin{aligned}
c^{(d(Y))}\left(f_{1}, f_{2}\right) & =\left(\int_{Y}\left(\left|\kappa^{(d(Y))}\left(\tau\left(f_{1}\right)\right)-\kappa^{(d(Y))}\left(\tau\left(f_{2}\right)\right)\right|\right)^{p}\right)^{1 / p} \\
& \rightarrow\left|\kappa^{(d(Y))}\left(\tau\left(f_{1}\right)\right)-\kappa^{(d(Y)}\left(\tau\left(f_{2}\right)\right)\right|, \text { as } p \rightarrow \infty .
\end{aligned}
$$

If $\gamma=d(Y), \kappa^{(\gamma)}(\tau(f))$ will be constant over the whole $Y$.

\subsection{Metrization Based Granulation}

The granulation technique is based on a construction, evolving from Urysohn's Lemma [9], that is used to prove the topological metrization theorem: A topology is metrizable if and only if it is $T_{1}$, regular, and has a $\sigma$-locally finite base.

Recall that any (pseudo-)metric space $(X, d)$ generates a topology $(X, \mathcal{T})$, where the open $r$-spheres $B(x, r)=\{y \in X \mid d(x, y)<r\}, x \in X, r>0$, form a base for $\mathcal{T}$. 
For the purpose of our constructions, we will assume the existence of a reasonably large set $\mathcal{F} \subseteq F(Y, M)$ so that each family

$$
\mathcal{B}_{r}=\left\{\tau^{-1}\left(B\left(g, \frac{1}{r}\right)\right) \mid g \in \mathcal{F} \subseteq F(Y, M)\right\}
$$

is locally finite and $B=\bigcup_{r \in N} \mathcal{B}_{r}$ is a $\sigma$-locally finite base for a topology on $F(X, L)$.

The metrics $d_{r, s}$ of $F(X, L)$ can now be defined as constructions in [8] related to proving the metrization theorem:

$$
d_{r, s}\left(f_{1}, f_{2}\right)=\sum_{U \in \mathcal{B}_{r}}\left|\varphi_{U}\left(f_{1}\right)-\varphi_{U}\left(f_{2}\right)\right|,
$$

where

$$
\varphi_{U}(f)=\left\{\begin{array}{l}
1, \text { if } f \in U^{\prime} \subseteq U \\
0, \text { if } f \notin U
\end{array}\right.
$$

and

$$
U^{\prime}=\bigcup_{V \in \mathcal{B}_{s}, \bar{V} \subseteq U} V .
$$

It can be shown that $\left(F(X, L), d_{r, s}\right)$ is a (pseudo-)metric space for all $r, s \in N$. We have $r \leq s$, and it is intuitively clear that the 'gap' between $U^{\prime}$ and the complement of $U$ closes up as $r$ approaches $s$. We will use $d^{(\gamma)}, \gamma=\frac{1}{r}$ to denote the intuitive counterpart of $d_{r, s}$, where the 'gap' between $U^{\prime}$ and the complement of $U$ is assumed to vanish or be negligible. Thus we arrive at our proposal for a metric on $F(X, L)$ :

$$
d^{(\gamma)}\left(f_{1}, f_{2}\right)=\operatorname{card}\left(B\left(\tau\left(f_{1}\right), \gamma\right) \cup B\left(\tau\left(f_{2}\right), \gamma\right) \sim B\left(\tau\left(f_{1}\right), \gamma\right) \cap B\left(\tau\left(f_{2}\right), \gamma\right)\right)
$$

where $\operatorname{card}(\cdot)$ is the cardinality for a set.

\subsection{Implementation}

The implementation of the $c^{(\gamma)}$-metric is rather straightforward and will not be treated in this section.

The implementation of the $d^{(\gamma)}$-metric is on the other hand more elaborated, this is because the idea of the metric is to count the number of functions $f \in$ $F(X, L)$ that, by the transformation $\tau$, is taken into either a surrounding of $\tau\left(f_{1}\right)$ or $\tau\left(f_{2}\right)$. The larger this set is the more $f_{1}$ and $f_{2}$ differ from each other. Of course, it is a non trivial task to actually implement this particular metric since we have no notion of how to generate the surrounding of a point $g \in F(Y, M)$ in the general practical case. This is due to the fact that each point $g$ is actually a 
function $g: Y \rightarrow M$ that in turn is implemented as a matrix. We can, however, calculate the distance between two points $g_{1}$ and $g_{2}$ with the $e_{p}$ metric and from this we are able to approximate the $d^{(\gamma)}$ metric according to:

$$
d^{(\gamma)}\left(f_{1}, f_{2}\right)=\left(\min \left\{\frac{e_{1}\left(\tau\left(f_{1}\right), \tau\left(f_{2}\right)\right)}{2 \gamma}, 1\right\}\right)^{p}
$$

The distance between $\tau\left(f_{1}\right)$ and $\tau\left(f_{2}\right)$ is related to the desired granularity $\gamma$. To raise the result to the power of $p$ approximates the dimensionality of the function space $F(Y, M)$.

Another approximation of $d^{(\gamma)}$ is

$$
d^{(\gamma)}\left(f_{1}, f_{2}\right)=\left\{\begin{array}{l}
1, \text { if } e_{p}\left(\tau\left(f_{1}\right), \tau\left(f_{2}\right)\right)>2 \gamma \\
1-\frac{2}{\pi}(\phi \sin \phi), \text { otherwise }
\end{array}\right.
$$

where $\phi=2 \arccos \left(\frac{e_{p}\left(\tau\left(f_{1}\right), \tau\left(f_{2}\right)\right)}{2 \gamma}\right)$. This is a fair approximation, provided we consider $B\left(\tau\left(f_{1}\right), \gamma\right)$ and $B\left(\tau\left(f_{2}\right), \gamma\right)$ as 2 -dimensional circles with radius $\gamma$. The distance is the non-overlapping area of the two circles normalized to the $[0,1]$ interval. If $B\left(\tau\left(f_{1}\right), \gamma\right)$ and $B\left(\tau\left(f_{2}\right), \gamma\right)$ are identical, there is no non-overlapping area and the distance is zero. If the distance between $\tau\left(f_{1}\right)$ and $\tau\left(f_{2}\right)$ is more than $2 \gamma$ there will be no overlapping area and $d^{(\gamma)}$ reaches its maximum value.

Of the suggested approximations of $d^{(\gamma)}$ the first is to prefer due to its generality and computational properties.

\section{The $\tau$-Transformation}

In our formal constructions, we see how each metric respectively are dependent on the transformations $\tau: F(X, L) \rightarrow F(Y, M)$. In the following subsections we will exemplify with specific choices for $\tau$.

There are a number of suitable $\tau$ :s that might be used. In this article we will concentrate on the SGLD as explained below. It is however stressed that basically any transform might be used. What transform to use depends on the application.

Spatial grey-level decencies (SGLD) were developed in 4 for handling statistical features of textures. We will use the corresponding image transformation as one of our case studies for $\tau$. However, we will use $\tau$ in a more general setting as compared to the original construction defined in 4 .

Let $X$ be the domain for the image, and let $L$ be the set of grey levels. As mentioned earlier an image is given as a function $f: X \rightarrow L$.

A pattern, corresponding to a texture, is given by $\pi: X \rightarrow X$, or generally by $\pi \subseteq X \times X$, or even $\pi \subseteq X^{n}$.

A notational convenience is given by $L^{2} \stackrel{\iota}{\rightarrow} F\left(L^{2},\{0,1\}\right)$, where 

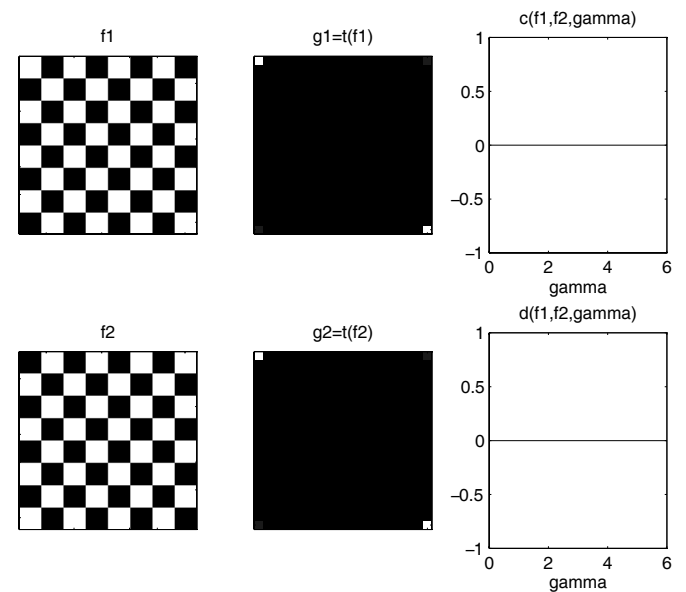

Fig. 2. The distances between the two chess boards textures. The distance is zero regardless the granularity used (right column). The SGLD-transform is shown in the center column.

$$
\iota\left(a_{1}, a_{2}\right)\left(l_{1}, l_{2}\right)=\left\{\begin{array}{l}
1, \text { if } a_{1}=l_{1}, a_{2}=l_{2}, \\
0, \text { otherwise }
\end{array}\right.
$$

Generalizing $\iota$ to involve not only crisp values, we have $L^{n} \stackrel{\iota}{\rightarrow} F\left(L^{n},[0,1]\right)$ where $\iota(\bar{a}): L^{n} \rightarrow[0,1]$ should satisfy $\iota(\bar{l})(\bar{l})=1$. This reveals the potentials of using $\iota$ in this way.

Given $\pi$ and $\iota$, we can now define the transformation $\tau=\tau(\pi, \iota): F(X, L) \rightarrow$ $F\left(L^{n}, R\right)$ through

$$
\tau(f)\left(a_{1}, \ldots, a_{n}\right)=\sum_{\left(x_{1}, \ldots, x_{n}\right) \in \pi} \iota\left(a_{1}, \ldots, a_{n}\right)\left(f\left(x_{1}\right), \ldots, f\left(x_{n}\right)\right) .
$$

Previously it was shown that two chess board patterns were given the maximum distance using the $d_{p}$-metric. By applying $d^{(\gamma)}$ and $c^{(\gamma)}$ this is avoided, see Figure 2, In this example is $\pi\left(x_{i, j}\right)=x_{i+1, j}$ used.

By introducing noise we disturb the statistical relationship between points in the texture. This implies that the textures that produced points in some SGLD transformation will produce smeared points when corrupted with noise, see Figure 3 The intensity around one of these points has the same distribution as the noise. Hence it can be seen that the noise in Figure 3 is of Gaussian type.

An example of a more general $\tau$ is to use the magnitude of the Fourier coefficients, that is $|\hat{f}|$. This representation is, however, not invariant under rotation, but by transforming $|\hat{f}|$ to a polar co-ordinate system the main direction of the texture is easily obtained. This is done since a main direction of the texture in 

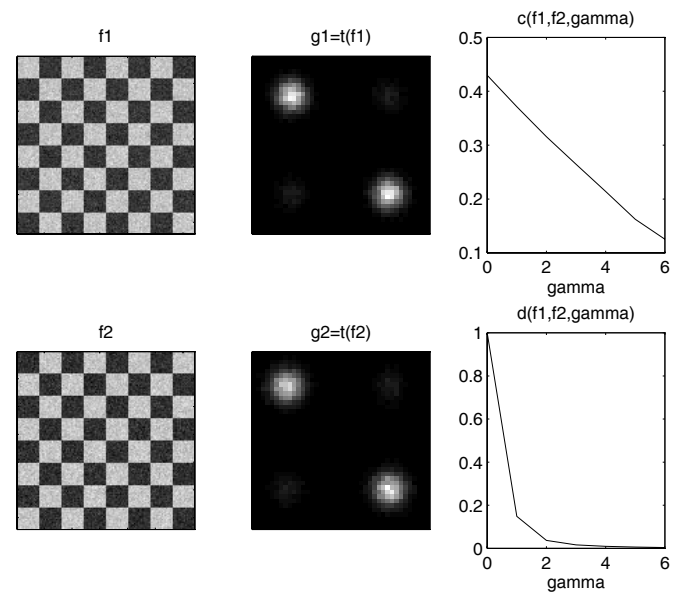

Fig. 3. The two chess boards textures corrupted with noise (left column). Note that the measured distance decreases with increased granularity (right column). The SGLDtransform is shown in the center column.

an image in the $F(X, L)$-domain generates a linear structure in the $F(Y, M)$ domain which, when in polar form clearly enhances the angle of the texture (or actually the angle orthogonal to the texture). Hence the energy as a function of texture angle is obtained as

$$
\alpha(\phi)=\int \hat{f}_{p}(\rho, \phi) d \rho
$$

where $\hat{f}_{p}(\rho, \phi)$ is the polar representation of $|\hat{f}|$. In the practical case this means that the two-dimensional image is reduced to an one-dimensional function. To obtain full rotational invariance it is possible to view $\alpha(\phi)$ as a periodic function with period $2 \pi$ and to define

$$
\bar{\alpha}(\phi):=\alpha(\phi-\mu)
$$

where $\mu$ satisfies

$$
\alpha(\mu)=\sup \alpha(\phi) .
$$

The definition in equation 22 ensures that $\bar{\alpha}(0)$ is the supremum of the energy function and hence it is meaningful to compare $\alpha$ 's by an ordinary $d_{p}$ metric.

\section{Results}

In this section properties of $c^{(\gamma)}$ and $d^{(\gamma)}$ will be further examined by some examples. The metrics are tested on four images shown in Figure 4 All images 


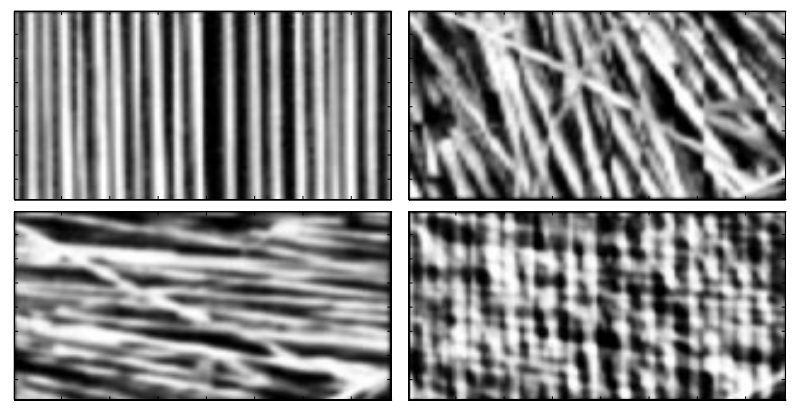

Fig. 4. Images $A$ and $B$ on top left respectively right and images $C$ and $D$ on bottom left and right respectively.

have the same mean values and variance of their pixel values. Image $D$ in Figure 4 is a combination of all the other images, $A$ to $C$. In tables [5, 6] and 7 the distances between noisy images are shown. The Gaussian noise is added so that the mean values of the images are preserved and the level of noise is $10 \%$ of the maximal pixel value. All images are $78 \times 156$ pixels.

The numerical value of the distance is somewhat irrelevant, since it is highly dependent on the normalization used. However, the 'close-far' relations, with corresponding ranking indicated in the tables, between the images does not change by normalization.

From table 3, it is clear that the $d_{p}$ metric taken on the original images does not separate the images at all. Hence, this metric will not be considered any more in this discussion.

The transformation used is a SGLD with $\pi\left(x_{i, j}\right)=x_{i, j+5}$ for all images. This transformation is most sensitive for textures in the vertical plane and hence is it most suitable for image $A$ in Figure 4. Given $\pi$, we expect the images $A$ and $C$ to be the most different. This is also the case for our metrics. Furthermore we expect $A$ and $B$ to be more similar than $B$ and $C$, since the main texture in $B$ is vertical while it is horizontal in $C$. However $d(A, B)<d(B, C)$ is not fulfilled by the $d_{1}$ metric.

It would be desirable that a metric preserves 'close-far' distances between images, even when they are corrupted with noise. From tables 56 and 7 it can be seen that $d^{(1)}$ preserves this order in a best way. This is expected since the $d^{(\gamma)}$-metric considers the whole range space when calculating the distances. This differs from the $d_{p^{-}}$and $c^{(\gamma)}$-metrics which consider point by point in the $Y$ space instead of the $F(Y, M)$ space.

The conclusion of these observations is that the proposed $c^{(\gamma)}$ - and $d^{(\gamma)}$-metric with a suitableSGLD-transform correspond to some extent to our cognitive sense of distance between images. 
Table 1. $c^{(2)}$-distances. Within parenthesis: the 'close-far'-rank.

\begin{tabular}{l|ccc}
$c^{(2)}$ & $\mathrm{B}$ & $\mathrm{C}$ & $\mathrm{D}$ \\
\hline $\mathrm{A}$ & $(2): 0.13(6): 0.24(3): 0.16$ \\
$\mathrm{~B}$ & - & $(5): 0.18$ & $(1): 0.05$ \\
$\mathrm{C}$ & - & - & $(4): 0.17$
\end{tabular}

Table 3. $d_{1}\left(f_{1}, f_{2}\right)$-distances.

\begin{tabular}{c|ccc}
$d_{1}$ & $\mathrm{~B}$ & $\mathrm{C}$ & $\mathrm{D}$ \\
\hline $\mathrm{A}$ & $(2): 0.30(2): 0.30(1): 0.20$ \\
$\mathrm{~B}$ & - & $(2): 0.30$ & $(1): 0.20$ \\
$\mathrm{C}$ & - & - & $(1): 0.20$
\end{tabular}

Table 5. $c^{(2)}\left(f_{1}, f_{2}\right)$-distances between noisy images.

\begin{tabular}{l|crc}
$c^{(2)}$ & $\mathrm{B}$ & $\mathrm{C}$ & $\mathrm{D}$ \\
\hline $\mathrm{A}$ & $(1): 0.25(4): 0.35(2): 0.26$ \\
$\mathrm{~B}$ & - & $(5): 0.38(3): 0.29$ \\
$\mathrm{C}$ & - & - & $(3): 0.29$
\end{tabular}

Table 2. $d^{(1)}$-distances.

\begin{tabular}{l|ccc}
$d^{(1)}$ & $\mathrm{B}$ & $\mathrm{C}$ & $\mathrm{D}$ \\
\hline $\mathrm{A}$ & $(3): 0.24(5): 0.30(2): 0.23$ \\
$\mathrm{~B}$ & - & $(4): 0.25(1): 0.015$ \\
$\mathrm{C}$ & - & - & $(4): 0.25$
\end{tabular}

Table 4. $d_{1}\left(\tau\left(f_{1}\right), \tau\left(f_{2}\right)\right)$-distances.

\begin{tabular}{c|ccc}
$d_{1}$ & $\mathrm{~B}$ & $\mathrm{C}$ & $\mathrm{D}$ \\
\hline $\mathrm{A}$ & $(4): 0.58(6): 0.75(5): 0.62$ \\
$\mathrm{~B}$ & - & $(3): 0.37$ & $(1): 0.13$ \\
$\mathrm{C}$ & - & - & $(2): 0.29$
\end{tabular}

Table 7. $d_{1}\left(\tau\left(f_{1}\right), \tau\left(f_{2}\right)\right)$-distances between noisy images.

$$
\begin{array}{l|ccc}
d_{1} & \mathrm{~B} & \mathrm{C} & \mathrm{D} \\
\hline \mathrm{A} & (4): 0.23(6): 0.59(2): 0.20 \\
\mathrm{~B} & - & (3): 0.21 & (1): 0.095 \\
\mathrm{C} & - & - & (5): 0.29
\end{array}
$$




\section{Conclusions and Further Work}

Metric image spaces are introduced with respect to corresponding image transformations. It is shown how a granulation can be introduced using neighbourhoods in domain and range image spaces, respectively. The metrics have complementary properties, and together they introduce a metrization typically useful in presence of transformations related to texture analysis.

Further work consists of finding better approximation of the $d^{(\gamma)}$-metric. We also need to add to our understanding what kind of overall texture information is represented by a given $g \in F(Y, M)$. In addition, the neighborhood of $g$ also needs an improved analytical description.

\section{References}

1. J. Serra. Image Analysis and Mathematical Morphology, volume 1. Academic Press, 1982.

2. D.L. Wilson, A.J. Baddeley, and R.A. Owens. A new metric for gray-scale image comparison. International Journal of Computer Vision, 24:5-17, 1997.

3. R. Lowen, and W. Peeters. On various classes of semi-pseudometrics used in pattern recognition. In Proc. Seventh IFSA World Congress, pages 232-237, 1997.

4. R.M. Haralick, K. Shanmugam, and I. Dinstein. Textural Features for Image Classification. IEEE Trans. Systems, man and Cybernetics, 3:610-621, 1973.

5. B. Sahiner, H.P. Chan, N. Petrick, M.A. Helvie, D.D. Adler, and M.M. Godsitt. Classification of Masses on Mammograms using Rubber-Band Straightening Transform and Feature Analysis. SPIE, proceedings series, 2710:44-50. 1996.

6. F. Georgsson, and S. Carlson, A Framework for Computer Aided Mammographic Screening for Breast Cancer, in N. Karssemeijer, M. Thijssen, J. Hendriks, and L. v. Erning (editors) Digital Mammography, Kluwer Academic Publishers, pages:415418, 1998.

7. J.F. Veenland, T.M. Link, W. Konermann, N. Meier, J.L. Grashuis, and E.S. Gelsema. Unraveling the role of structure and density in determining vertebral bone strength. Calcified Tissue Int, (61):474-479, 1997.

8. J.L. Kelley. General Topology. Van Nostrand, New York, 1955.

9. P. Urysohn. Über die Machtigheit der Zusammenhägen Mengen. Math. Ann., 94:262-295, 1925. 